\title{
Evaluation of Quadratus Lumborum Block after laparoscopic cholecystectomy
}

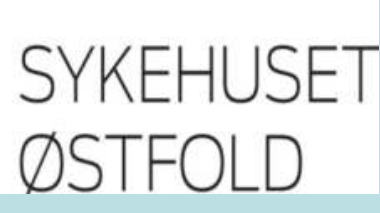

\author{
J.S. Vamnes ${ }^{1}$, M. Sörenstua ${ }^{1}$, K.I. Solbakk¹, B. Sterud ${ }^{1}$, A.C. Linqvist Leonardsen ${ }^{2}$ \\ Östfold Hospital Trust, Department of Anesthesia, Moss, Norway.
}

${ }^{2}$ Östfold University College, Faculty of Health and Welfare, Fredrikstad, Norway.

\section{Introduction:}

Enhanced recovery after surgery and laparoscopic approach are essential for ambulatory surgery. Many patients have nausea and vomiting postoperatively, and pain is an expected but undesirable effect after surgery. We strive for a postoperative period without pain and nausea, thus facilitating early discharge. Quadratus Lumborum Block (QLB) is a recommended multimodal method of reducing postoperative pain in laparoscopic and open surgery (Murouchi et al 2016). We utilize the transmuscular block (QLB-3). The aim of the study is to find out if a bilateral QLB has beneficial analgesic and antiemetic effects after laparoscopic cholecystectomy versus traditional oral and/or intravenous (iv) analgesia.

\section{Methods:}

After approval by the Regional Committee for Medical and Health Research Ethics (2017/1894) and Clinical Trials, 75 patients scheduled for ambulatory laparoscopic cholecystectomy were randomly selected to receive one of the following: 1) Group QLB: Ropivacaine 3,75 mg/ml, $20 \mathrm{ml}$ on each side preoperatively, 2) Placebo group QLB: Isotonic saline, $20 \mathrm{ml}$ on each side preoperatively, and 3) Control group: Parenteral and oral analgesics postoperatively.

The surgeons applied postoperative subcutaneous wound infiltration to all patients with $20 \mathrm{ml}$ ropivacaine $2 \mathrm{mg} / \mathrm{ml}$. The maximum allowed dose was $3 \mathrm{mg} / \mathrm{kg}$ body weight (BW). If $B W<65 \mathrm{~kg}$, we reduced the concentration, using the same volume. All three groups received necessary analgesics po or iv. Anesthesia was induced and maintained with propofol, remifentanil and oxycodone. Rescue analgesics (tramadol and oxycodone) were given postoperatively. All patients were discharged 2 - 3 hours postoperatively. Tramadol, or a combination of paracetamol and codeine, were used as analgesics at home. Use of analgesics and pain (Numeric Rating Scale (NRS) 0 - 10) were recorded during stay in the recovery room, and obtained through phone contact 24,48 and 168 hours postoperatively.

The number of patients required for the study has been calculated on the basis of opioid consumption. The minimum difference of interest is set to $20 \%$, based on a clinical evaluation. Assuming an $a=0,05$, sample size calculations showed a need for 69 patients (23 in each group) included, to achieve a power of $80 \%(\beta=0.2)$.

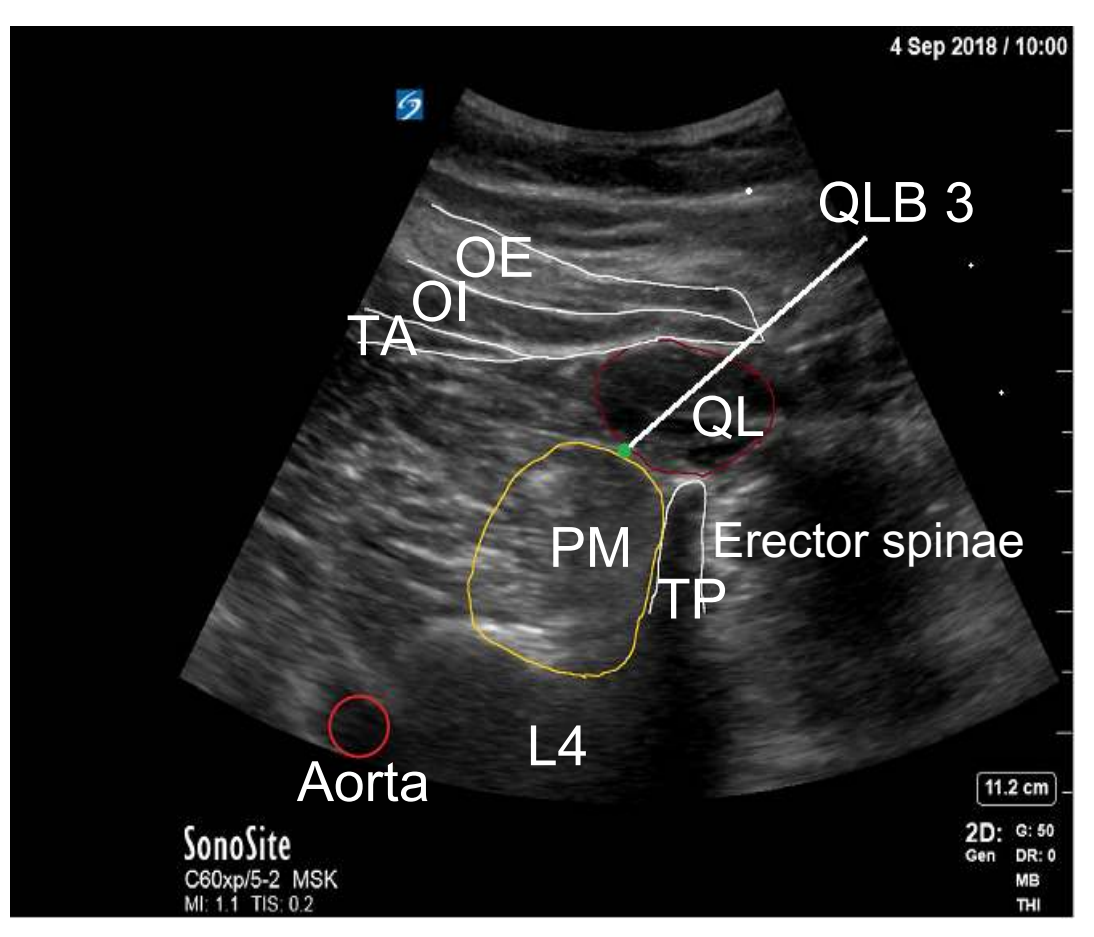

Transmuscular anterior OLB

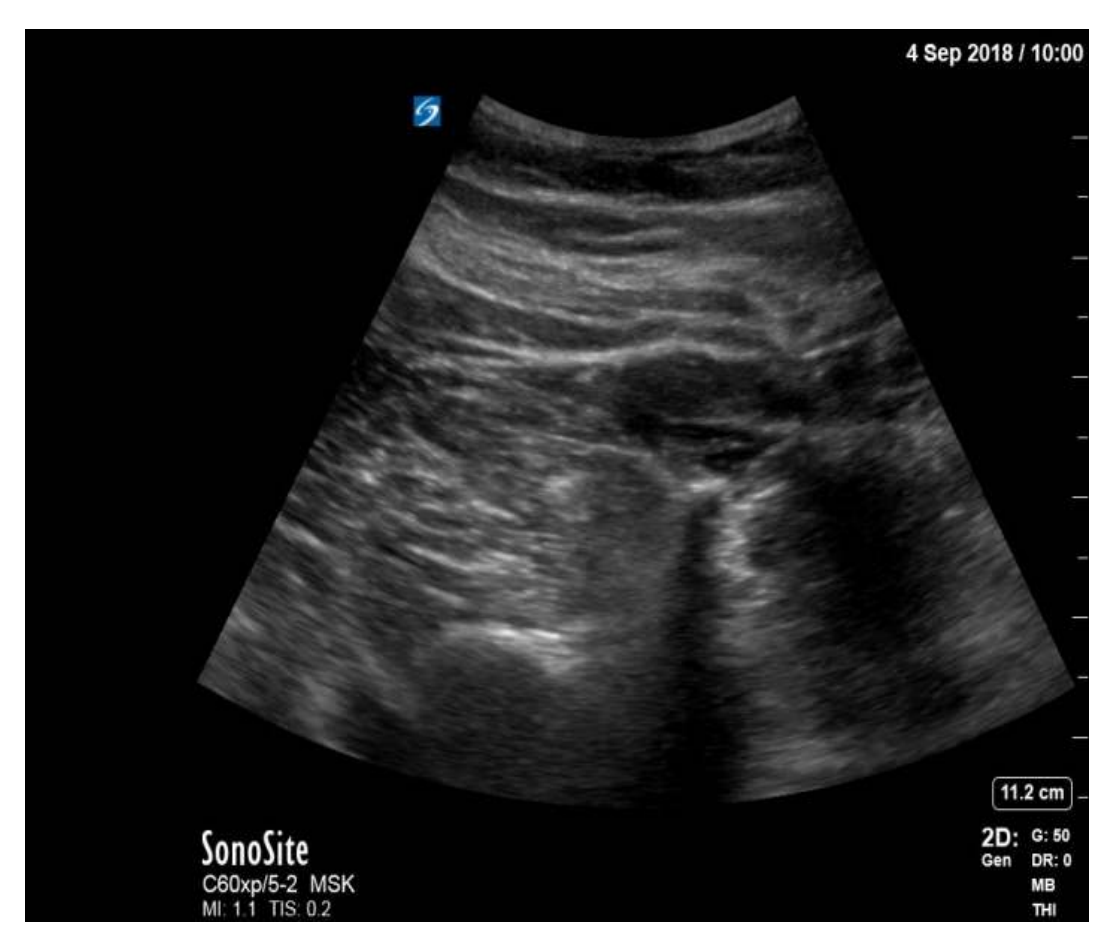

Probe 5-2 $\mathrm{MHz}$ Curved

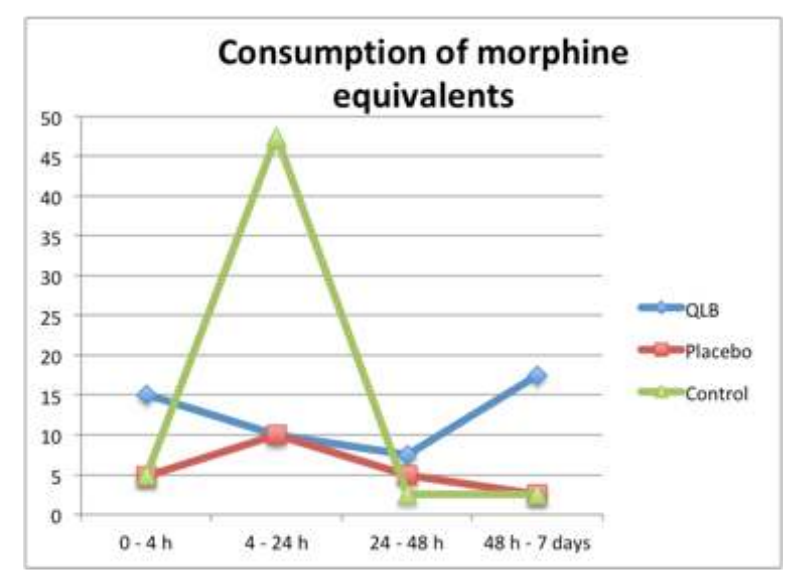

Pain score (NRS 0 - 10)

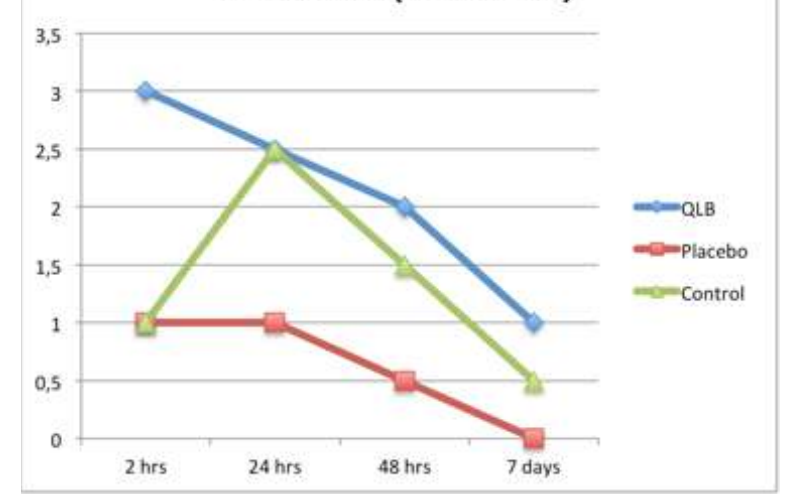

\section{Results:}

Duration of surgery (mean $36 \mathrm{~min}$ ) and intraoperative consumption of analgesics were similar in all groups. The consumption of rescue analgesics in the recovery room was similar in all groups. The consumption of oral morphine equivalents and the NRS at 0-24 h, 24-48 h and 48 -168 h, showed no statistical differences between the groups. The QLB group had significantly less incidents of nausea than both the control- and placebo group at all measure points during the first 24 hours.

\section{Discussion}

Limitations in this study is mainly the short postoperative stay of 2-3 hours. However, this is a day-case study conducted according to our clinical routines. The short stay reduces the collection of data during the time for the assumed effect of the QLB. In this setting with minimal invasive surgery, and the fact that our satisfied patients prior to this study were sent home without any nerve blocks, one may assume small differences between the treatment groups. Most of the studies of these nerve blocks have focused primarily on endpoints such as pain and consumption of analgesics. Here we demonstrate significant differences concerning nausea, but were not able to show differences concerning pain. Informal discussions with recovery nurses during the data analysis revealed that although the nurses were blinded there appeared to be patients who were more capable to dress without help, had less nausea and ate and drank more than other patients. Recovery is a complex process, and when we only monitor the NRS and opioid consumption, we might miss factors that are important for the patients' total outcome. The quality of recovery could be better evaluated with a more differentiated validated recovery score such as the QoR-15 form.

\section{Conclusion:}

In our study, quadratus lumborum block with ropivacaine has a significant effect on reducing nausea during the first 24 hours after surgery. Reduction of pain and opioid consumption was similar in all groups in our setting. A validated Recovery Score, such as the Quality of Recovery-15 items (QoR-15) (Stark, Myles, Burke) may reveal further benefits of a QLB.

Ref:

1. Quadratus Lumborum Block: Analgesic Effects and Chronological Ropivacaine Concentrations After Laparoscopic Surgery

Murouchi T, Iwasaki S, Yamakage M. Reg Anesth Pain Med. 2016 Mar-Apr;41(2):146-50

2. Development and Psychometric Evaluation of a Postoperative Quality of Recovery Score. The QoR-15. Stark PA, Myles PS, Bruke JA.: Anesthesiology $118 ; 1332-1340$ 